\title{
Bouncing on the fringes of the Dental System: Clinical Dental Technicians, a decade after their creation
}

\author{
Gurveer Jaggee BChD MSc \\ Community Dental Officer \\ (Former Dental Core Trainee and Honorary Research Associate, King's College London Dental \\ Institute) \\ Email gjaggee@hotmail.com \\ Jayne Dooey BDS \\ Dental Core Trainee \\ St George's Hospital \\ (Former Dental Core Trainee and Honorary Research Associate, King's College London Dental \\ Institute) \\ Email Jaynedooey13@aol.com \\ Professor Jennifer E Gallagher MBE \\ Dean for International Affairs \\ Head of Population and Patient Health \\ Newland-Pedley Professor of Oral Health Strategy \\ Honorary Consultant in Dental Public Health \\ King's College London Dental Institute at Guy's, King's College \& St Thomas' Hospitals \\ Denmark Hill Campus. Bessemer Road, London SE5 9RS \\ Telephone 02032995171/3481 \\ Email: jenny.gallagher@kcl.ac.uk \\ David R Radford* BDS PhD FDS MRD \\ Reader in Integrated Dental Education and Multi-professional Care \\ King's College London Dental Institute at Guy's, King's College \& St Thomas' Hospitals \\ St Thomas' Street, London Bridge, London SE1 9RT \\ and the University of Portsmouth Dental Academy, Faculty of Science, University of Portsmouth. \\ Portsmouth PO1 2QG \\ Email david.radford@kcl.ac.uk
}

* Corresponding author

Key words: workforce, clinical dental technician, scope of practice, profession.

\footnotetext{
Abstract

Aim To investigate Clinical Dental Technicians (CDTs) current working practices and their perception as registered dental professionals regarding their practice of dentistry, role and relationships in the
} 
dental team and within the UK healthcare system. Methods A postal questionnaire was sent to all 304 CDTs registered with the United Kingdom General Dental Council (September 2015), which included an information sheet and a consent form. Data were entered onto computer and analysed using SPSS and free-text responses were analysed using thematic analysis. Results A response rate of $37.8 \%$ was achieved. The majority of participants were male (92\%), based in England (83\%) working full time $(66.4 \%)$, in the private sector $(89.4 \%)$ and spending up to $50 \%$ of their time working as a CDT carrying out clinical work. CDTs reported barriers to their progression as the lack of an NHS contract, and their limited scope of practice which they wished to have extended to further their skill-set and include direct access to provide partial dentures without a dentist's prescription. Conclusion There was some evidence that CDTs are becoming more recognised and valued members of the dental team. Whilst CDTs felt they had progressed since their establishment in 2007, the respondents considered there was still a need for progress to be made of integration within the dental system and recognition by the public. CDTs wished to see changes to their performer status and to have a wider scope of practice. The majority of CDTs work in the private sector thus there is a need to explore their future roles within the state dental provision.

\section{In Brief Box}

- A follow up study into the current status of CDTs in the UK

- CDTs appear more confident as independent clinical providers of complete dentures and are still expressing a wish to expand their scope of practice

- Most CDTs perceived/reported their relationship with dentists as symbiotic for both parties

- The respondent CDTs expressed very strong but mixed opinions regarding working as part of the NHS 


\section{INTRODUCTION}

Clinical Dental Technicians (CDTs) are an established group of Dental Care Professionals (DCPs) in the United Kingdom (UK), recognised by the UK General Dental Council (GDC) as a separate professional group since 2007. ${ }^{1}$ Ninety-two CDTs were registered with the GDC by March 2009. By September 2015 this had increased to 304 and by January 2017, further increased to 352 registrants. The decision to create CDTs as part of Professions complementary to dentistry, ${ }^{2}$ was in response to the Nuffield Foundation report on the Education and training of personal auxiliary to dentistry, acting as a catalyst for the formal recognition of CDTs. ${ }^{3}$

The GDC clearly define the roles and limitations of CDTs in the guidance; Scope of Practice. ${ }^{4}$ It states that CDTs should have undergone initial training as dental technicians and then further training in order to prescribe and provide complete dentures direct to patients and provide and fit other dental devices chnician. Currently, the Faculty of General Dental Practice at the Royal College of Surgeons England and the Royal College of Surgeons of Edinburgh both award a Diploma in Clinical Dental Technology. There have been three providers of the education leading to this qualification, the Edinburgh Postgraduate Dental Institute, Health Education England (Kent, Surrey and Sussex Postgraduate Deanery) and the University of Central Lancashire. In order to be eligible for these programmes all applicants must have a qualification as a dental technician and be registered with the GDC. ${ }^{1}$

Prior to CDTs recognition and registration with the GDC, Ross et al. ${ }^{5}$ in 2007 reported a positive response to the prospect of statutory registration. Subsequently Leyssen et al. ${ }^{6}$ in 2013 investigated the experiences of CDTs. Results from that study found that CDTs were "embracing their new status as an occupational group within dentistry". ${ }^{6}$ However, there was an inability to provide NHS care due to status within the healthcare system and financial remuneration. Thus, it is important to investigate their experiences now as a more established profession, in order to assess whether their formation has proven to be beneficial to the dental team, healthcare system and CDTs themselves.

Therefore, the aims of this study were first, to investigate registered CDTs current working patterns, patient base and scope of practice. Second, to explore their perception of their current roles and relationships within the dental team and their perceptions of the public's awareness of their role. Third, and last, their opinion was sought regarding their status as a professional group and any changes that they would feel would be beneficial to their scope of practice. 
A cross sectional postal questionnaire survey was sent to all registered CDTs compiled from the Dental Care Professionals (DCP) register recorded on the GDC website. At the start of the study in September 2015, there was a population of 304 registered CDTs. A questionnaire survey, consent form and an information sheet were posted to all CDT registrants in September 2015, inviting participation. The information sheet included a description of the research being undertaken including the purpose of the study and how it would affect the participant. The questionnaire survey was based on the initial study conducted by Leyssen et $a l^{6}$ which included 30 questions divided into four sections relating to CDTs work patterns, patient base, working relationships and future growth as a professional group. To increase the response rate an approach was used which involved sending follow up reminder letters. ${ }^{7}$ One hundred and fifteen questionnaires were returned; quantitative data were analysed descriptively using SPSS. The qualitative aspects of the questionnaire were analysed using a framework methodology ${ }^{8}$ and key themes were identified.

\section{RESULTS}

Of the 304 registered CDTs who were sent the questionnaire, there were 115 responses (37.8\%). Of these, 113 questionnaires were usable representing $37.2 \%$ of the total professional group. The majority of respondents were male $(93 \%, n=104)$ and working in England $(83 \%, n=94)$ (Table 1), with the largest category aged $45-54$ years $(42.5 \%, n=48)$.

\section{Working pattern}

Most participants reported working fulltime $(66.4 \%, n=75)$, with less than a third working part time $(27.4 \%, n=31)$ and a small proportion flexibly, working a mixture of part time and full time $(6.2 \%$, $\mathrm{n}=7$ ) (Table 1). This ranged from working 1-7 days per week, with the largest proportion of respondents $(28.3 \%, n=32)$ working five days per week.

All CDTs spent some of their working hours utilising their clinical skills combined with technical work, with only eight respondents $(7.1 \%, n=8)$ spending no time carrying out their own laboratory work. Forty respondents carried out no technical work for dentists $(35.4 \%, n=40)$. The highest estimated percentage of treatment carried out was in the provision of complete dentures (50\%) with only $20 \%$ of time being spent providing partial acrylic dentures and metal based partial dentures, with $10 \%$ of time making 'other prostheses'. Whilst $41.6 \%(n=47)$ undertook their own dental laboratory work, $53.1 \%(n=60)$ utilised dental technicians for completion of certain technical aspects of denture provision.

\section{Patient base}


CDTs reported treating patients from 45 years of age upwards, identifying the largest age-group as 70-74-year-olds $(61.1 \%, n=69)$. The volume of patients per week ranged from 1-137; however, most CDTs reported seeing an average of 25 patients per week. When asked about patients they would not treat, the majority of CDTs stated they would treat all patients $(57.5 \%, n=65)$; however, a minority would not treat patients in retirement homes/domiciliary visits $(18.6 \%, n=21)$, or those with complex medical histories $(8.8 \%, n=10)$, and dementia $(1.8 \%, n=2)$.

Of the 113 participating CDTs, 89.4\% ( $n=101)$ worked privately, $1.8 \%(n=2)$ for private insurance, 4.4\% $(n=5)$ a combination of NHS and private and $6.2 \%(n=7)$ solely for the NHS. One third of respondents $32 \%$ ( $n=36$ ) stated that the main barrier to providing work on the NHS was the lack of a contract.

\section{Relationship with the public}

The respondents reported that they felt that the role of a CDT was not well understood by the public and indeed by some dental practitioners. Whilst there was some understanding that a CDT was able to provide dentures, there was confusion when it came to partially dentate patients requiring examination and care-planning by a dentist first. In order to gain patients, $90.3 \%$ of CDTs ( $n=102)$ relied on word of mouth, followed by newspaper advertisements $(65.5 \%, n=74)$ and $59.3 \%(n=67)$ on referrals from dentists. The average number of referrals to participants, from dentists, was eleven per month.

\section{Relationship with dentists}

In addition to the above, more than half reported working with a dentist $(55.8 \%, n=63)$, with the significant majority of respondents $(87.6 \%, n=99)$ working with $1-5$ dentists. Only $2.7 \%(n=3)$ reported working with more than 10 dentists. A significant percentage of CDTs $(40.7 \%, n=46)$ stated that they had positive experiences and working relationships with dentists. There is further evidence of their integration within the dental team in delivering pathways of care, with $92 \%$ ( $n=104)$ of CDTs reporting referral of patients to dentists for reasons other than treatment for partial dentures. The respondents stated that most referrals were for further dental treatment $(46.9 \%$, $\mathrm{n}=53$ ), whilst a number of CDTs reported that they had referred patients for a second opinion on suspicious lesions $(14.7 \% n=13)$ and $23 \%$ ( $n$ 26) for implant assessment. Over half of CDTs $(54.9 \%$ $\mathrm{n}=62$ ) had not referred a patient to a dentist for the construction of a complete denture within the last two years.

\section{Thematic analysis}


Three main themes were identified from the free-hand responses. They were: A) Finding a professional position within the dental team; B) NHS Involvement; and, C) Enhanced scope of practice. Each theme is illustrated with quotations in the following format: Participant Number, Age range, Gender, Date of registration.

\section{A) Finding a professional position within the dental team}

Most CDTs reportedly perceived their relationship with dentists as symbiotic for both parties. They felt that once the dentist saw the standard of work they produced, they were able to recognise and utilise their CDTs skills and were advocates of working together as a team. Respondents felt that dentists were generally happy to have CDTs provide denture work. This was due to a mixture of reasons including: dentists' lack of understanding of technical aspects; disinterest in providing dentures; CDTs being seen as a valued member of the team; and the need for collaboration between dentists and CDTs, due to the reducing number of edentulous patients but often with greater presenting difficulties.

- I feel it is working well for me. I like dentists to check partial cases and refer on to me. [49, 55-64, M, 2013]

- Some dentists encourage all members of the dental team and appreciate enthusiasm and technical input. Others are precious about position and don't agree CDTs should be undertaking work. All dentists are different. [33, 35-44, M, 2012]

Whilst CDTs reported to be happy to continue working with dentists, they differed however, in their opinion of the ideal set up. Most liked the idea of having a dentist attending a few days a week at their own CDT practice, whereas others were happy to go to a dental practice. CDTs felt that their understanding of the technical aspects of denture construction allowed them to produce a better calibre of prosthetic work compared to dentists who may be limited by providing care funded by the NHS.

- The NHS fee for dentures does not equate to the time and costs involved in the examination consultation and manufacturing process. [48, 55-64, M, 2014]

- The quality of work that is produced by a CDT practice is normally vastly superior to NHS dentures. $[29,55-64, \mathrm{M}, 2007]$

Due to the increased life expectancy in the UK, ${ }^{9}$ many had a positive outlook on CDTs developing further within the team and becoming more recognised in denture provision for older people. The perceived decreased competency amongst young dentists was also seen as an opportunity for 
clinical dental technicians and that younger dentists were happy to refer denture work to CDTs. They felt that the younger dentists were being trained to recognise CDTs as part of the dental team.

- As the population grows older more dentures will be needed. Also younger more recently qualified dentists will come into profession with little or no interest and understanding of dentures, consequently CDTs role should become stronger. [27, 45-54, M, 2011]

- The UK requires more foot soldiers in the field, to serve the aging population. The CDT is a critical part of the team that will allow other areas of dental care to run more efficiently and to focus on patient satisfaction and provision of other treatments. [55, 45-54, M, 2007]

However, some CDTs reported experiencing hostility from some dentists who were sceptical about their role and what they could provide. Some were disillusioned and negative about future progression.

- Not really sure, as a lab owner I struggle to see a future for me as a CDT. My hope was that dentists would refer patients to the practice set up within the lab, but very few appear willing to do this. [78, 45-54, M, 2013]

- Not sure at this point, seems like a need for CDTs but not sure NHS will train or want to train in the future. [91, 35-44, M, 2011]

\section{NHS Involvement}

The respondent CDTs expressed very strong but mixed opinions regarding working as part of the NHS. Some felt that it would not allow them to produce the best quality work causing a drop in standards. Others were more willing to work as part of the NHS. However, there was a recognition of the need for an NHS option for those patients struggling to pay privately for CDT care.

- The UDA system (sic Units of Dental Activity) does not work for P/P or F/F (sic Partial/partial dentures or Complete/complete dentures). Too much work involved to produce an acceptable standard at price paid. Labs should have standards and be paid direct from NHS. [32, 35-44, $M, 2012]$

- I am not aware CDTs can obtain a NHS contract directly. This barrier needs to be removed to allow for improved patient care, particularly domiciliary care. [55, 45-54, M, 2013]

There was recognition amongst some respondents that there is a need for the NHS to embrace CDTs and provide and ensure NHS dentures are reimbursed appropriately.

- if dentists cannot make it pay how can we? Lowering of time and standards would be unacceptable. $[47,<65, \mathrm{M}, 2003]$ 
- The NHS fee that a dentist receives for NHS work is inadequate (too small). This should be increased and the lab fee should come from/be paid directly to the labs. This will prevent delayed lab fee payment. [39, 35-44 M, 2014]

Other concerns included a perceived lack of independence, restrictions and administrative overload.

- For a very small practice like mine, the administration would be quite difficult, also we have enough business without taking on NHS work. [95, 55-64, M, 2010]

\section{Enhanced Scope of Practice}

The aspiration to change their scope of practice to widen direct access was very apparent. The main desire was to have independence in the provision of partial dentures. Some CDTs felt that with added training, they should have the opportunity to prepare teeth for removable partial dentures, provide mouth guards without a prescription, and be able to take impressions for repairs.

- Improved/further training to allow for direct access where applicable to reduce the burden on the NHS. This is a must as the present system is failing patients daily! [55, 45-54, M, 2013]

- Provision of partial dentures without treatment plan or patient with a treatment plan and sent from their dentist. Teeth whitening, adding composite or rest seats to natural teeth. [68, 35-44, M, 2007]

- Direct access to allow certain treatments, additions, impressions for repairs, unscrewing temporary abutments to allow try-ins. [38, 45-54, M, 2007]

\section{DISCUSSION}

Despite the findings of this follow-up cross-sectional survey revealing similar patterns of working and perceived barriers, some differences have been noted since the work of Leyssen et al. ${ }^{6}$ conducted eight years ago. A lower proportion of CDTs appear to be carrying out technical work for dentists, spending more time on their own cases and over half of the respondent cohort of CDTs had not referred a patient for the provision of a complete denture to a dentist in two years, compared to the $44 \%$ who had done so in the previous study. ${ }^{6}$ There has been an increase in the number of referrals received from dentists per month, on average 11 per month compared to 1-6 reported from $2009 .{ }^{6}$ Respondents were predominantly from England, male and aged between 45-44 years. It is unknown whether this reflects the age or gender distribution of the profession but they certainly, overall, were positive in their outlook and on furthering the care they offer to patients. Despite their motivation to progress clinically, the majority of respondents split their time between clinical and technical work and saw on average 25 patients per week. Possible reasons for this may include: CDTs limiting their work to ensure a positive life balance; patients not being prepared to pay private fees 
for care; or, their working as originally envisaged undertaking both the clinical and technical work for patients. A further reason for this work pattern was that they enjoyed undertaking the technical aspects as originally trained to do and wished to preserve those skills. Qualitative results from Leyssen et al., ${ }^{6}$ revealed that some CDTs felt it was a part of their job description to carry out technical work for dentists; however, 35\% of respondents reported not undertaking any technical work for dentists, thus, potentially depriving dentists of access to some of the most experienced dental prosthetic technicians. There was an aspiration to widen their scope of practice to enable direct access, specifically in relation to the provision of partial dentures, and other aspects of prosthodontic care. The study by Ross, et al. ${ }^{5}$ prior to registration with the GDC, reported that $84 \%$ of respondents felt that expansion of the CDT role was probable in the future.

The majority of CDTs worked privately, primarily as they are not currently considered for performer status by the NHS, although this is similar to other DCPs (hygienist-therapists, dental hygienists) and second, they felt that the NHS fees were insufficient for the level of care they provide. Subsequently, by remaining within the private sector, CDTs are in direct competition with dentists, which could account for some of the hostility between the two professions. It also means that their services are limited to a smaller proportion of the population able to afford private treatment. With regard to the aspiration of some CDTs to be able to provide care as NHS performers, there are mixed views on adding DCPs to performer lists.

The main patient base reported by CDTs was in the 70-74-year age-bracket, compared with the original study's results of $64-69$ years. ${ }^{6}$ The Adult Dental Health Survey ${ }^{10}$ (ADHS) has reported that $6 \%$ of adults in the UK are edentate, however broken down to age groups, $5 \%$ of 55 to 64 -year olds and $47 \%$ over 85 were edentulous. Although the encouraging headline figure of $6 \%$ of UK adults being edentulous is widely quoted, that still equates to 2.7 million adults in the UK, as well as glossing over the need for demised dentitions to be supported by a combination of single complete dentures and partial dentures, which is equally, or arguably more complex care.

Forecasting future need is problematic due to a reduction in tooth loss being counter balanced by an aging population. However, as younger dentists gain less experience in denture provision during their undergraduate education and have fewer opportunities to practice the necessary care planning and removable prosthodontic skills, ${ }^{11,12}$ there will be a continued demand for dental team members with prosthetic expertise. By recognising CDTs as an asset to the dental team, positive working relationships should be established together with an increase in the effectiveness in the delivery of 
services. ${ }^{13}$ The $A D H S^{10}$ also reported the significantly larger percentage of adults aged 65 years and over wearing partial dentures. Of these, $16 \%$ are from manual and routine occupational households compared to $8 \%$ from managerial and professional households. Only one third of patients aged 55 years and over, reported that their last course of treatment was paid for privately. For the ageing population who may not be able to afford private dental care there should ideally in future be access to NHS clinical dental technician services with adequate geographic coverage. It has already been suggested that hygienist-therapists may be ideally positioned to contribute to the delivery of periodontal and restorative care for older people ${ }^{14}$ so a model of care, incorporating a multidisciplinary approach of appropriate DCPs including CDTs is attractive to cope with the challenges for our aging population with multiple co-morbidities. Thus, we argue that CDTs should be embraced by the NHS system, where they wish to engage, rather than bouncing on the edge of the system.

Limitations of the study include the low response rate to the postal questionnaire, with only a third of the registered CDTs in the UK participating in the study. The response rate was similar to our previous study in $2013 .{ }^{6}$ This could be due to a number of factors: lack of engagement as is common with questionnaire style studies, the timing of the survey and perceived relevance of the subject; and, possible scepticism towards the dental profession, recognising that the survey team were dentists. The expansion of scope of practice to increase the CDT skill mix could aid integration with the dental team to better serve patients certainly with the increasing age of the population. Further research is required to explore the implications for further training if their scope of practice is to be enhanced, particularly to include direct access for partial dentures, preparation of rest seat and guide planes for partial dentures, and, provision of implant supported complete dentures.

\section{CONCLUSION}

Within the limitations of the study there was evidence that CDTs are becoming more recognised and valued members of the dental team. Clinical dental technicians have increased in number since their registration with the GDC as a profession complimentary to dentistry in 2007 . Whilst CDTs felt they had progressed since their establishment as a registered group, the participants considered there was still a need for progress before being fully accepted within the dental team and recognised by the public. CDTs wished to see changes to their performer status and to have a wider scope of practice. Whilst there has been some improvement in their development as a member of dental team, the majority of CDTs work in the private sector; thus, there is a need to explore their future roles within the state dental provision particularly for older people. 


\section{Acknowledgements}

We would like to thank the participants for their involvement and Sarah Curtis, Research Associate Population and Patient Health Divison, King's College London Dental Institute and Paul Hellyer for help with the preparation of the manuscript.

\section{References}

1) The General Dental Council. Clinical Dental Technology, Programmes and qualifications. (2017). from https://www.gdc-uk.org/professionals/education/recentinspections/inspections-clinical-dt (Accessed 12 November 2017).

2) The General Dental Council. Professions Complementary to Dentistry. Regulations Order of Council 2006 No. 1440 from http://www.legislation.gov.uk/uksi/2006/1440/resources. (Accessed 4 October 2017).

3) Grace M. The Nuffield Report. Br Dent J 1993; 175: 231.

4) The General Dental Council. "Scope of Practice. 2013. gdc-uk. org/Newsandpublications/Publications/Publications/Scope\% 20of\% 20Practice\% 20September. from file:///C:/Users/k1653867/Documents/Literature/Scope\%20of\%20Practice\%20September\% 202013.pdf. (Accessed 12 November 2017).

5) Ross M K, Ibbetson R J, Turner S. Activity and education of clinical dental technicians: a UK survey. Br Dent J 2007; 203: E22.

6) Leyssen W, Clark RKF, Gallagher JE, Radford DR. Developing professional status: an investigation into the working patterns, working relationships and vision for the future of UK clinical dental technicians. Br Dent J 2013; 214: E3.

7) Dillman DA, Smyth JD, Christian LM. Internet, phone, mail, and mixed-mode surveys: the tailored design method. $4^{\text {thed }}$ Chichester.John Wiley \& Sons. 2014. 
8) Richie J,Spencer L, O'Connor W. Carrying out qualitative research. In Richie J, Lewis J McNaughton C et al (eds), Qualatitative research practice: a guide for social science students and researchers. London: Sage 2014.

9) Office for National Statistics (2017). "National life tables, UK: 2014 to 2016.", from https://www.ons.gov.uk/peoplepopulationandcommunity/birthsdeathsandmarriages/lifeex pectancies/bulletins/nationallifetablesunitedkingdom/2014to2016. (Accessed 12 Novemeber 2017).

10) White DA, Tsakos G, Pitts NB, Fuller E, Douglas GVA, Murray JJ, Steele JG. Adult Dental Health Survey 2009: common oral health conditions and their impact on the population. $\mathrm{Br}$ Dent J 2012; 213: 567-572. DOI: 10.1038/sj.bdj.2012.1088.

11) Clark, RKF, Radford DR, Juszczyk AS. Current trends in complete denture teaching in British dental schools. Br Dent J 2010; 208: E10.

12) Clark, RKF, Radford DR, Juszczyk AS. Current trends in removable partial denture teaching in British dental schools. Br Dent J 2011; 211: 531-535.

13) Borrill $C$, West $M$, Shapiro $D$, Rees $D$. Team working and effectiveness in health care. $B r J$ Healthcare Mang 2000; 6: 364-371.

14) Hellyer PH. The older dental patient-who cares? Br Dent J 2011; 211: 109-110. 
Table 1 Demographics and working patterns of respondents

\begin{tabular}{|l|l|l|l|}
\hline & & Number (113*) & Percentage \\
\hline Sex & Male & 105 & 92.9 \\
\hline & Female & 7 & 6.2 \\
\hline Country work place & En-declared & 1 & 0.9 \\
\hline & Scotland & 94 & 83.2 \\
\hline & Wales & 9 & 8.0 \\
\hline & Northern Ireland & 3 & 4.4 \\
\hline & Channel Island & 1 & 2.7 \\
\hline Working pattern & Un-declared & 1 & 0.9 \\
\hline & Full-time & 75 & 0.9 \\
\hline & Part-time & 31 & 66.4 \\
\hline & Flexible employment & 7 & 27.4 \\
\hline
\end{tabular}

*Number of useable returned questionnaires 